\title{
Correction to: Emerging Topics in the Behavioral Neuroscience of Tinnitus
}

\author{
Grant D. Searchfield, Jinsheng Zhang, Roshni Biswas, Dirk De Ridder, \\ Brian Deutsch, Deborah A. Hall, Sylvie Hébert, Tobias Kleinjung, \\ Maria Kleinstäuber, Berthold Langguth, Jose Antonio Lopez-Escamez, \\ Michael R. D. Maslin, Birgit Mazurek, Jay F. Piccirillo, Richard Salvi, \\ Winfried Schlee, Abraham Shulman, Susan Shore, Agnieszka J. Szczepek, \\ Paul F. Smith, Sarah M. Theodoroff, Dunja Vajsakovic, Cornelia Weise, \\ and Yiwen Zheng
}

\section{Correction to:}

Chapter "Emerging Topics in the Behavioral

Neuroscience of Tinnitus" in:

Grant D. Searchfield et al., Curr Topics Behav Neurosci, https://doi.org/10.1007/7854_2020_217

The original version of this chapter unfortunately contained two errors: in author name and order of author. These two errors has been corrected and the below are the updated correction:

1. The author name "Sylvie Hall Hébert" is changed to "Sylvie Hébert".

2. The order of author name Deborah A. Hall is listed before Sylvie Hébert.

\footnotetext{
The updated online version of this chapter can be found at
} https://doi.org/10.1007/7854_2020_217 